\title{
Wind-induced deformations in a segmented mirror
}

\author{
Stephen Padin
}

\begin{abstract}
A Zernike expansion of wind-induced deformations in a segmented mirror is described. The wind model is a frozen turbulent field with a Kolmogorov spectrum for scales smaller than the outer scale and a flat spectrum for scales larger than the outer scale. The approach allows a mode-by-mode comparison of the wave-front error contributions from atmospheric phase distortions, wind-induced deformations, and the mirror control system noise. This is used to design a controller that minimizes the mirror surface errors by application of corrections based on edge sensor measurements and wave-front measurements on a guide star. (C) 2002 Optical Society of America
\end{abstract}

OCIS codes: $\quad 010.1080,010.1330,010.7060,220.1000$.

\section{Introduction}

The optical performance of a telescope mirror is degraded by wind-induced deformations (Ref. 1 and references therein). These deformations are caused by pressure fluctuations at the mirror surface and by wind-induced structural vibrations coupling to the mirror. Structural vibrations depend on the design details of the telescope, so in the following analysis I consider just the direct interaction between the wind and the mirror. The approach focuses on the statistical properties of the wind rather than on the details of the airflow around the mirror, and the control discussion assumes a segmented mirror. The segmented mirrors in the Keck telescopes, and the mirror proposed for the California Extremely Large Telescope (CELT), use capacitive edge sensors to measure the relative heights of the segments. ${ }^{2,3}$ The edge sensors are sensitive to mirror modes with feature sizes of the order of the size of a segment, but the sensitivity to low-order modes, with much larger features, is poor. This causes the mirror control system to amplify the edge sensor noise added to the low-order modes and is a serious problem for a mirror with a large number of segments. The proposed solution to this for the CELT is to use edge sensor measurements to correct the high-order mirror modes and wave-front measurements on a guide star

S. Padin (spadin@caltech.edu) is with the California Institute of Technology, MS 105-24, 1200 E. California Boulevard, Pasadena, California 91125.

Received 1 November 2001; revised manuscript received 29 January 2002.

0003-6935/02/132381-09\$15.00/0

(C) 2002 Optical Society of America to correct the low-order modes..$^{3,4}$ The primary role of the wave-front sensor is to measure the dc component of low-order gravitational and thermal deformations, but it can also measure low-order wind-induced deformations and atmospheric phase distortions.

The control requirements for the mirror depend on the type of instrument being used with the telescope. For observations with extreme adaptive optics systems, wind-induced deformations can be corrected along with ground-layer atmospheric phase distortions, so there is no reason to adjust the figure of the telescope mirror, except perhaps for high-order modes that are difficult for an adaptive optics system to correct. For single-conjugate adaptive optics systems, systems with a limited actuator travel in the adaptive mirrors, and for seeing-limited observations in the case of large wind-induced deformations, it may be advantageous to minimize the mirror surface errors. This involves one having to compromise among correcting deformations caused by the wind, adding unwanted distortions from the atmosphere for corrections based on wave-front measurements, and adding noise from the mirror control system for corrections based on edge sensor measurements. These effects are estimated in Sections 2-4, and the mirror control strategy is discussed in Section 5.

\section{Atmospheric Phase Distortions}

The Zernike expansion of atmospheric phase distortions follows Noll ${ }^{5}$ and Roddier. ${ }^{6-8}$ The analysis includes the decorrelation of atmospheric Zernike modes away from a guide star and the time variation of the modes that is due to a frozen screen of turbulence drifting in front of the telescope. The results allow us to estimate the wave-front error that is 
added to the target when the mirror is adjusted by wave-front measurements on a guide star.

The atmospheric phase distortion at time $t$ and normalized position $\mathbf{r}$ in the aperture is

$$
\varphi\left(\mathbf{r}-\frac{\mathbf{v}}{R} t\right)=\sum_{j} a_{j}\left(\frac{\mathbf{v}}{R} t\right) Z_{j}(\mathbf{r})
$$

where $\mathbf{v}$ is the wind velocity, $R$ is the mirror radius, and $a_{j}$ is the coefficient of the $j$ th modified Zernike polynomial $Z_{j}$. Then

$$
a_{j}\left(\frac{\mathbf{v}}{R} t\right)=\int W(r) Z_{j}(\mathbf{r}) \varphi\left(\mathbf{r}-\frac{\mathbf{v}}{R} t\right) \mathrm{d} \mathbf{r},
$$

where $r=|\mathbf{r}|, W(r)=1 / \pi$ for $r \leq 1$, and $W(r)=0$ for $r>1$. Because $a_{j}(\mathbf{r})$ is the convolution of $\varphi(\mathbf{r})$ with $W(r) Z_{j}(\mathbf{r})$, the spatial power spectrum of $a_{j}(\mathbf{r})$ is

$$
\Phi_{j}(\mathbf{k})=\Phi(k)\left|\mathrm{FT}\left[W(r) Z_{j}(\mathbf{r})\right]\right|^{2}=\Phi(k)\left|Q_{j}(\mathbf{k})\right|^{2},
$$

where

$$
\Phi(k)=0.023\left(\frac{R}{r_{0}}\right)^{5 / 3} k^{-11 / 3}
$$

is the spatial power spectrum of $\varphi(\mathbf{r})$ for Kolmogorov turbulence, ${ }^{5,9} r_{0}$ is the Fried parameter, ${ }^{10}$ and

$$
\begin{aligned}
Q_{j}(\mathbf{k})= & \sqrt{n+1} \frac{\mathrm{J}_{n+1}(2 \pi k)}{\pi k} \\
& \times\left\{\begin{array}{ll}
(-1)^{(n-m) / 2} \sqrt{2} \cos m \phi & \text { even } j \\
(-1)^{(n-m) / 2} \sqrt{2} \sin m \phi & \text { odd } j \\
(-1)^{n / 2}(m=0) &
\end{array}\right\}
\end{aligned}
$$

is the Fourier transform $(\mathrm{FT})$ of $W(r) Z_{j}(\mathbf{r})$, where $\mathbf{k}=$ $k \exp (i \phi)$, and $m$ and $n$ are the azimuthal frequency and radial degree of the $j$ th Zernike mode. The spatial frequency $k$ does not include $2 \pi$ terms, i.e., $W(r) Z_{j}(\mathbf{r})=\int Q_{j}(\mathbf{k}) \exp (i 2 \pi k r) \mathrm{d} \mathbf{k}$.

The angular dependence of the $j$ th atmospheric Zernike mode is given by the spatial covariance of $a_{j}(\mathbf{r})$ :

$$
B_{j}(\mathbf{\rho})=\left\langle a_{j}(\mathbf{r}) a_{j}(\mathbf{r}+\boldsymbol{\rho})\right\rangle=\mathrm{FT}\left[\Phi_{j}(\mathbf{k})\right],
$$

where $\boldsymbol{\rho}$ is the normalized lateral displacement of a frozen turbulent screen. For a thin screen at height $h,|\boldsymbol{\rho}|=h \theta / R \cos \gamma$, where $\gamma$ is the zenith angle and $\theta$ is the angle between the guide star and the target. If we choose $\boldsymbol{\rho}=(\rho, 0)$, then

$$
\begin{aligned}
B_{j}(\rho)= & 0.023\left(\frac{R}{r_{0}}\right)^{5 / 3} \frac{n+1}{\pi^{2}} \int_{-\infty}^{\infty} \int_{-\infty}^{\infty} k^{-17 / 3}\left[\mathrm{~J}_{n+1}(2 \pi k)\right]^{2} \\
& \times \exp \left(i 2 \pi \rho k_{x}\right)\left\{\begin{array}{ll}
2 \cos ^{2} m \phi & \text { even } j \\
2 \sin ^{2} m \phi & \text { odd } j \\
1(m=0) &
\end{array}\right\} \mathrm{d} k_{x} \mathrm{~d} k_{y},
\end{aligned}
$$

where $\left(k_{x}, k_{y}\right)=\mathbf{k}$. Equation (7) is divergent at small $k$ for the $n=0$ (piston) mode, and the $n=1, j=$ 2 and 3 (tip and tilt) modes also have high power at small $k$. These difficulties are the result of the $-11 / 3$ power-law spectrum in Eq. (4) being applied to all scales, but in the real atmosphere Eq. (4) is valid only for distances up to the outer scale $L_{0}$. For larger scales, the frozen screen assumption breaks down and the power spectrum flattens. If $L_{0} \gg R$, we can estimate the effect of the outer scale by forcing the power spectrum of atmospheric phase distortions to be flat for scales larger than $L_{0}$. We use

$$
\Phi(k)=0.023\left(\frac{R}{r_{0}}\right)^{5 / 3} \frac{1}{k^{11 / 3}+\left(R / L_{0}\right)^{11 / 3}},
$$

which is similar to the von Karman spectrum, ${ }^{11,12}$ but has a sharper transition between the outer scale and the inertial regions. The spatial covariance of $a_{j}(\mathbf{r})$ is then

$$
\begin{aligned}
B_{j}(\rho)= & 0.023\left(\frac{R}{r_{0}}\right)^{5 / 3} \frac{n+1}{\pi^{2}} \int_{-\infty}^{\infty} \int_{-\infty}^{\infty} \\
& \times \frac{\left[\mathrm{J}_{n+1}(2 \pi k)\right]^{2} \exp \left(i 2 \pi \rho k_{x}\right)}{\left[k^{11 / 3}+\left(R / L_{0}\right)^{11 / 3}\right] k^{2}} \\
& \times \begin{cases}2 \cos ^{2} m \phi & \text { even } j \\
2 \sin ^{2} m \phi & \text { odd } j \\
1(m=0) & \mathrm{d}\left(m k_{x} \mathrm{~d} k_{y} .\right.\end{cases}
\end{aligned}
$$

The uncorrected wave-front variance in the direction of the target, in the $j$ th mode, is $B_{j}(0)-B_{j}(\rho)$; and because the Zernike modes are nearly independent for $n>1$ (Ref. 13), the wave-front variance in the $n$th radial degree is

$$
\left\langle\sigma_{\theta}^{2}(\rho, n)\right\rangle=\sum_{j}\left[B_{j}(0)-B_{j}(\rho)\right],
$$

where the summation runs over the $n+1$ azimuthal modes in the $n$th radial degree, i.e., $[(n+1) n+$ $2] / 2 \leq j \leq[(n+3) n+2] / 2$. Then

$$
\left\langle\sigma_{\theta}^{2}(\rho, n)\right\rangle=\left[1-\Gamma_{n}(\rho)\right] \sum_{j} B_{j}(0),
$$

where

$$
\Gamma_{n}(\rho)=\frac{\sum_{j} B_{j}(\rho)}{\sum_{j} B_{j}(0)}
$$

is the correlation coefficient between the wave-front variances in the directions of the guide star and the target. Because $B_{j}(\rho)$ is real, we need consider only 
the cosine term in the exponent in Eq. (9); then for $\rho$ $\ll 1$,

$$
\begin{aligned}
1-\Gamma_{n}(\rho)= & 2 \pi^{2} \rho^{2} \\
& \times \frac{\int_{-\infty}^{\infty} \int_{-\infty}^{\infty} \frac{k_{x}^{2}\left[\mathrm{~J}_{n+1}(2 \pi k)\right]^{2}}{\left[k^{11 / 3}+\left(R / L_{0}\right)^{11 / 3}\right] k^{2}} \mathrm{~d} k_{x} \mathrm{~d} k_{y}}{\int_{-\infty}^{\infty} \int_{-\infty}^{\infty} \frac{\left[\mathrm{J}_{n+1}(2 \pi k)\right]^{2}}{\left[k^{11 / 3}+\left(R / L_{0}\right)^{11 / 3}\right] k^{2}} \mathrm{~d} k_{x} \mathrm{~d} k_{y}} .
\end{aligned}
$$

Figure 1 shows $\left[1-\Gamma_{n}(\rho)\right] / \rho^{2}$ for $1 \leq n \leq 50$ and $L_{0}=$ 10R. For $\rho \ll 1,\left[1-\Gamma_{n}(\rho)\right] \sim \rho^{2} n^{5 / 3}$.

For a frozen screen, a temporal displacement $\tau$ is equivalent to a spatial displacement $v \tau / R$, so the temporal covariance of $a_{j}(t)$ is

$$
\begin{aligned}
C_{j}(\tau) & =\left\langle a_{j}(t) a_{j}(t+\tau)\right\rangle=\left\langle a_{j}(\mathbf{r}) a_{j}\left(\mathbf{r}+\frac{\mathbf{v} \tau}{R}\right)\right\rangle \\
& =\mathrm{FT}\left[\Phi_{j}(\mathbf{k})\right] .
\end{aligned}
$$

If we choose $\mathbf{v}=(v, 0)$, then

$$
\begin{aligned}
C_{j}(\tau) & =\int_{-\infty}^{\infty} \int_{-\infty}^{\infty} \Phi_{j}\left(k_{x}, k_{y}\right) \exp \left(i 2 \pi \frac{v}{R} \tau k_{x}\right) \mathrm{d} k_{x} \mathrm{~d} k_{y} \\
& =\int_{-\infty}^{\infty} \int_{-\infty}^{\infty} \Phi_{j}\left(\frac{f R}{v}, k_{y}\right) \exp (i 2 \pi f \tau) \frac{R}{v} \mathrm{~d} f \mathrm{~d} k_{y},
\end{aligned}
$$

where $f=k_{x} v / R$ is the frequency with which the wind crosses distance $R / k_{x}$. The temporal frequency spectrum of $a_{j}(t)$ is

$$
F_{j}\left(\frac{f R}{v}\right)=\mathrm{FT}\left[C_{j}\left(\frac{\tau v}{R}\right)\right]=\int_{-\infty}^{\infty} \Phi_{j}\left(\frac{f R}{v}, k_{y}\right) \mathrm{d} k_{y}
$$

and when we use Eqs. (3) and (8),

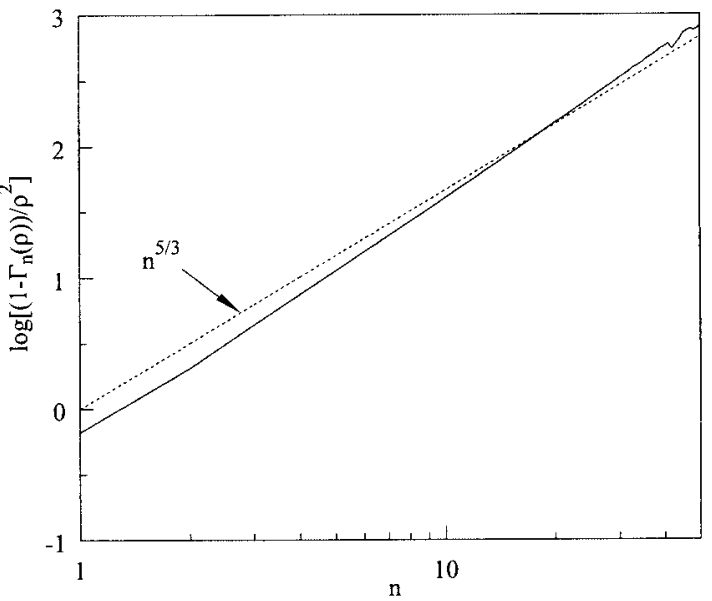

Fig. 1. Spatial decorrelation of the wave front for small offsets between the target and the guide star and $L_{0}=10 R$ [Eq. (13)]. $\Gamma_{n}(\rho)$ is the correlation coefficient between the wave-front variances in two directions offset by $\rho=h \theta / R \cos \gamma$, where $\theta$ is the angle between the two directions, $h$ is the height of the turbulent atmospheric layer, $R$ is the mirror radius, and $\gamma$ is the zenith angle.

where $[(n+1) n+2] / 2 \leq j \leq[(n+3) n+2] / 2$. This is shown in Fig. 2 for $1 \leq n \leq 50$ and $L_{0}=10 R$. All the modes have flat spectra at frequencies much less than the wind-crossing frequency for the mirror, so $\left\langle\sigma_{\tau}{ }^{2}\left(\tau_{\text {int }} \gg R / v\right)\right\rangle \propto 1 / \tau_{\text {int }}$. The sharp cutoff at $\tau_{\text {int }}$ used in Eq. (18) is convenient for the estimation of $\left\langle\sigma_{\tau}^{2}\left(\tau_{\text {int }}, n\right)\right\rangle$, but the control system transfer function $T(f)$ will usually be more complicated. Then

$$
\left\langle\sigma_{\tau}^{2}\left(\tau_{\mathrm{int}}, n\right)\right\rangle=2 \sum_{j} \int_{0}^{\infty} T\left(\frac{f R}{v}\right) F_{j}\left(\frac{f R}{v}\right) \mathrm{d} \frac{f R}{v} .
$$

The fraction of $\left\langle\sigma_{\tau}{ }^{2}\left(\tau_{\text {int }}, n\right)\right\rangle$ that is uncorrelated between the guide star and the target is

$$
\begin{aligned}
\left\langle\sigma_{\text {atm }}^{2}\left(\tau_{\text {int }}, \rho, n\right)\right\rangle= & \left\langle\sigma_{\tau}^{2}\left(\tau_{\text {int }}, n\right)\right\rangle\left[1-\Gamma_{n}(\rho)\right] \\
& \sim\left\langle\sigma_{\tau}^{2}\left(\tau_{\text {int }}, n\right)\right\rangle \rho^{2} n^{5 / 3} \text { for } \rho \ll 1 .
\end{aligned}
$$

$$
\begin{aligned}
F_{j}\left(\frac{f R}{v}\right)= & 0.023\left(\frac{R}{r_{0}}\right)^{5 / 3} \frac{n+1}{\pi^{2}} \int_{-\infty}^{\infty} \frac{\left(\mathrm{J}_{n+1}\left\{2 \pi\left[(f R / v)^{2}+k_{y}{ }^{2}\right]^{1 / 2}\right\}\right)^{2}}{\left\{\left[(f R / v)^{2}+k_{y}{ }^{2}\right]^{11 / 6}+\left(R / L_{0}\right)^{11 / 3}\right\}\left[(f R / v)^{2}+k_{y}{ }^{2}\right]} \\
& \times\left\{\begin{array}{ll}
2 \cos ^{2} m \phi & \text { even } j \\
2 \sin ^{2} m \phi & \text { odd } j \\
1(m=0)
\end{array}\right\} \mathrm{d} k_{y} .
\end{aligned}
$$

If wave-front measurements on the guide star are used to correct the mirror on time scales longer than the measurement integration time $\tau_{\text {int }}$, then the variance of the correction for the $n$th radial degree is

$$
\left\langle\sigma_{\tau}^{2}\left(\tau_{\mathrm{int}}, n\right)\right\rangle=2 \sum_{j} \int_{0}^{R / v \tau_{\mathrm{int}}} F_{j}\left(\frac{f R}{v}\right) \mathrm{d} \frac{f R}{v},
$$

This is the wave-front error that is added to the target when the mirror is adjusted by use of wave-front measurements on the guide star.

\section{Wind-Induced Deformations}

If the mirror is in the inertial region, we can apply a frozen turbulent field model to estimate the wave- 


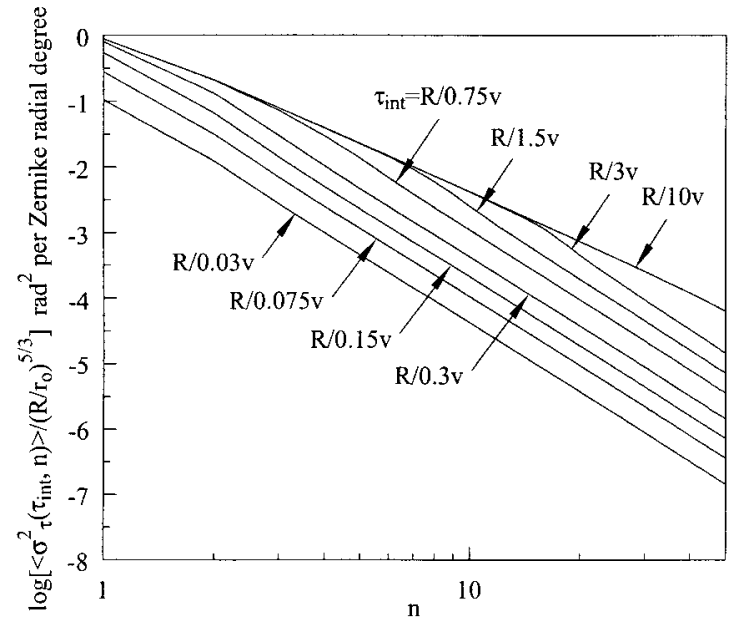

Fig. 2. Wave-front variance on the guide star on time scales longer than $\tau_{\text {int }}$ for turbulence with $L_{0}=10 R$ [Eq. (18)].

front error that is due to pressure fluctuations at the mirror surface. This is particularly straightforward for a segmented mirror in a stiff cell, because for modes with features larger than a segment, the openloop stiffness per unit area is determined primarily by the mirror actuators and is independent of the scale size of the mode features. The frozen turbulence model is crude, because although it gives a reasonable estimate of the pressure fluctuations in an unperturbed field, it does not properly account for the effect of the mirror on the airflow.

In a turbulent atmosphere, a fluctuation of scale size $l$ passes an observer in time $T \sim l / u$, where $u$ is the mean wind speed. The time for the fluctuation to evolve appreciably is $\tau \sim l / s$, where $s$ is the internal velocity in the fluctuation. The mean rate of energy dissipation, per unit mass, is $\varepsilon \sim$ (1/ $2) \varsigma^{2}(\varsigma / l)$, so $\varepsilon l \sim \varsigma^{3}$ and $\tau \sim l^{2 / 3} / \varepsilon^{1 / 3}$. But $\varepsilon L_{0} \sim u^{3}$, where $L_{0}$ is the outer scale, so $T \sim L_{0}^{2 / 3} / \varepsilon^{1 / 3}$ and $\tau / T \sim\left(l / L_{0}\right)^{2 / 3}$. If $l \ll L_{0}, \tau \ll T$, and the fluctuation does not evolve as it passes the observer. In this case the turbulence is frozen and the velocity field has a one-dimensional spatial power spectrum proportional to $k^{-5 / 3}$ and a temporal spectrum proportional to $f^{-5 / 3}$ (Ref. 9). Near the ground, $L_{0}$ is typically of the order of the height above the ground, so the primary mirror of a telescope in a large dome is likely to be in, or near, the inertial region. The mirror and telescope tube will tend to break up large-scale structures in the turbulence, and this moves energy to smaller spatial scales and reduces $L_{0}$. For $l>L_{0}$, the power spectra flatten, and spatial and temporal displacements of the field are no longer equivalent, but the spatial and temporal scales are still correlated. If $L_{0}$ is not much smaller than $R$, we can use the approach of Section 2 to make a rough estimate of the effect of the outer scale by forcing the power spectrum of pressure fluctuations to be flat for $l>L_{0}$. This model will tend to underestimate the temporal frequency of changes for $l>L_{0}$, and if $L_{0} \sim R$, the first few Zernike radial degrees will be affected.

In the inertial region, the structure function for the velocity field is $D_{u}(r)=u^{2} f\left(r / l_{0}\right)$, where $l_{0}=\left(v^{3} / \varepsilon\right)^{1 / 4}$ is the inner scale, $u=(\varepsilon v)^{1 / 4}$, and $v$ is the viscosity. $D$ has the dimensions of velocity squared and is independent of viscosity, so $f\left(r / l_{0}\right) \propto\left(r / l_{0}\right)^{2 / 3}$ and $D_{u}(r)$ $\propto \varepsilon^{2 / 3} r^{2 / 3}$, which is the Kolmogorov 2/3 law. Applying a similar argument to the structure function for $u^{2}$ yields $D_{u^{2}}(r) \propto r^{4 / 3}$. The one-dimensional spatial power spectrum is then ${ }^{9}$

$$
\begin{aligned}
V(k) & \propto \frac{1}{k} \int_{0}^{\infty} D_{u^{2}}^{\prime}(r) \sin (2 \pi k r) \mathrm{d} r \\
& \propto \frac{1}{k} \int_{0}^{\infty} r^{1 / 3} \sin (2 \pi k r) \mathrm{d} r \propto k^{-7 / 3},
\end{aligned}
$$

and the corresponding three-dimensional spatial power spectrum is

$$
\Omega(k) \propto \frac{1}{k} V^{\prime}(k) \propto k^{-13 / 3}
$$

In the following discussion, we first consider a frozen field moving normal to the mirror, and then we consider a frozen screen moving across the mirror surface, as in the model for the atmospheric Zernike mode expansion in Section 2. The spatial power spectrum for pressure fluctuations, and hence for mirror deformations, follows a $k^{-13 / 3}$ law in the inertial region; when we use this in Eq. (3), the power spectrum of the wave-front error for the $j$ th Zernike mode for a normal-incidence wind model is

$$
\begin{aligned}
\Theta_{j}\left(k_{x}, k_{y}, k_{z}\right)= & \frac{A}{\left(k_{x}{ }^{2}+k_{y}{ }^{2}+k_{z}{ }^{2}\right)^{13 / 6}+\left(R / L_{m}\right)^{13 / 3}} \\
& \times\left|Q_{j}\left(k_{x}, k_{y}\right)\right|^{2},
\end{aligned}
$$

where $A$ is a constant and $L_{m}$ is the outer scale of the turbulence at the mirror surface. The $z$ direction in Eq. (23) is parallel to the optical axis and the wind velocity. Following the approach of Section 2, the temporal frequency spectrum of the $j$ th Zernike coefficient of the wave-front error is

$$
\Psi_{j}\left(\frac{f R}{u}\right)=\int_{-\infty}^{\infty} \int_{-\infty}^{\infty} \Theta_{j}\left(k_{x}, k_{y}, \frac{f R}{u}\right) \mathrm{d} k_{x} \mathrm{~d} k_{y},
$$

where $u$ is the wind speed normal to the mirror surface and $f=k_{z} u / R$ is the frequency with which the wind crosses distance $R / k_{z}$. $\Theta_{j}(\mathbf{k})$ is circularly symmetric about the $z$ axis, so we can sum the $n+1$ azimuthal modes in the $n$th radial degree. Then 


$$
\begin{aligned}
\psi_{n}\left(\frac{f R}{u}\right) & =\int_{-\infty}^{\infty} \int_{-\infty}^{\infty}\left(\frac{n+1}{\pi}\right)^{2} \frac{A\left\{\mathrm{~J}_{n+1}\left[2 \pi\left(k_{x}{ }^{2}+k_{y}{ }^{2}\right)^{1 / 2}\right]\right\}^{2}}{\left\{\left[k_{x}{ }^{2}+k_{y}{ }^{2}+(f R / u)^{2}\right]^{13 / 6}+\left(R / L_{m}\right)^{13 / 3}\right\}\left(k_{x}{ }^{2}+k_{y}{ }^{2}\right)} \mathrm{d} k_{x} \mathrm{~d} k_{y} \\
& =\int_{0}^{\infty}\left(\frac{n+1}{\pi}\right)^{2} \frac{A\left[\mathrm{~J}_{n+1}(2 \pi \xi)\right]^{2}}{\left\{\left[\xi^{2}+(f R / u)^{2}\right]^{13 / 6}+\left(R / L_{m}\right)^{13 / 3}\right\} \xi^{2}} 2 \pi \xi \mathrm{d} \xi .
\end{aligned}
$$

The rms pressure fluctuation at the mirror surface is $2 \rho_{\text {air }} u \sigma_{u}$, where $\rho_{\text {air }}$ is the density of air and $u$ and $\sigma_{u}$ are the mean and rms wind speeds normal to the mirror surface. The corresponding rms deformation in the mirror is $2 \rho_{\text {air }} u \sigma_{u} A_{\text {seg }} / K$, where $A_{\text {seg }}$ is the segment area per actuator and $K$ is the actuator stiffness $\left(\mathrm{Nm}^{-1}\right)$. The wave-front variance that is due to wind-induced deformations is then

$$
\left\langle\sigma_{w}{ }^{2}\right\rangle=\left(2 \frac{2 \pi}{\lambda} \frac{A_{\mathrm{seg}}}{K} 2 \rho_{\mathrm{air}} u \sigma_{u}\right)^{2},
$$

where $\lambda$ is the wavelength. By use of Eq. (25),

$$
\left\langle\sigma_{w}{ }^{2}\right\rangle=2 \sum_{n} \int_{f_{m} R / u}^{\infty} \psi_{n}\left(\frac{f R}{u}\right) \mathrm{d} \frac{f R}{u},
$$

where $f_{m}$ is the low-frequency limit for measurements of $u$ and $\sigma_{u}$ in Eq. (26). Equations (26) and (27) allow us to find the constant $A$, given measurements of the wind velocity spectrum, if $L_{m}$ and $f_{m}$ are the same for both the model and the wind measurements. The highest radial degree in the summation in Eq. (27) is determined by the number of independent modes in the mirror. If the mirror has $M$ actuators, there are $M$ independent modes, and the summation must include all the corresponding Zernike modes. The Zernike modes are nearly independent, and there are $n+1$ azimuthal modes in the $n$th radial degree, so the summation runs to degree $N$, where $[(N+1)(N+$ $2)] / 2 \sim M$. Because the power in the higher-order modes is small, the variance calculation is insensitive to the choice of $N$.

Wind-induced deformations in the mirror on time scales longer than $\tau_{\text {int }}$ are included in measurements of the wave-front error on the guide star and are corrected. Deformations on time scales shorter than $\tau_{\text {int }}$ remain incorrected, and their contribution to the wave-front variance in the $n$th radial degree is

$$
\left\langle\sigma_{\text {wind }}^{2}\left(\tau_{\text {int }}, n\right)\right\rangle=2 \int_{R / u \tau_{\text {int }}}^{\infty} \Psi_{n}\left(\frac{f R}{u}\right) \mathrm{d} \frac{f R}{u} .
$$

Figure 3 shows $\left\langle\sigma_{\text {wind }}{ }^{2}\left(\tau_{\text {int }}, n\right)\right\rangle /\left\langle{\sigma_{w}}^{2}\right\rangle$ for $1 \leq n \leq 50$ and $L_{m}=R$, with $\left\langle\sigma_{\mathrm{w}}{ }^{2}\right\rangle$ calculated for the frequency range $0.1 u / R \leq f \leq 20 u / R$ and radial degrees $1 \leq n \leq$ 50 . This assumes that the wind measurements exclude the $n=0$ (piston) mode, which can be achieved if we take the difference between two velocity sensors separated by the mirror diameter. $L_{m}=R$ is an estimate based on measurements of the temporal power spectrum of pressure fluctuations at the mirror surface of the southern Gemini telescope. ${ }^{14}$ A sub- stantial reduction in the wave-front variance in a particular mode requires corrections on time scales shorter than the wind crossing time for a mode feature; so for a given $\tau_{\text {int }}$, wind-induced deformations with a low radial degree are better corrected. If the control system transfer function is $T(f)$ [see Eq. (19)], Eq. (28) becomes

$$
\left\langle\sigma_{\text {wind }}^{2}\left(\tau_{\text {int }}, n\right)\right\rangle=2 \int_{0}^{\infty}\left[1-T\left(\frac{f R}{u}\right)\right] \Psi_{n}\left(\frac{f R}{u}\right) \mathrm{d} \frac{f R}{u} .
$$

The finite stiffness of the mirror cell may significantly increase deformations in the low-order modes. This can be modeled as a mode-dependent stiffness $K(n)$; because deformations in the mirror are inversely proportional to stiffnes, the wave-front variance is

$\left\langle{\sigma_{\text {wind }}}^{2}\left[\tau_{\text {int }}, K(n), n\right]\right\rangle=\left[\frac{K}{K(n)}\right]^{2}\left\langle\sigma_{\text {wind }}^{2}\left(\tau_{\text {int }}, n\right)\right\rangle$.

For a simply supported plate of radius $a$, the stiffness is proportional to $1 / a^{2}$ (Ref. 15). Deformations with Zernike radial degree $n$ have features with scale size $\sim a / n$, so the stiffness for plate modes in the $n$th radial degree is roughly proportional to $n^{2}$. If the mirror cell behaves like a plate, $K(n) \propto n^{2}$; and be-

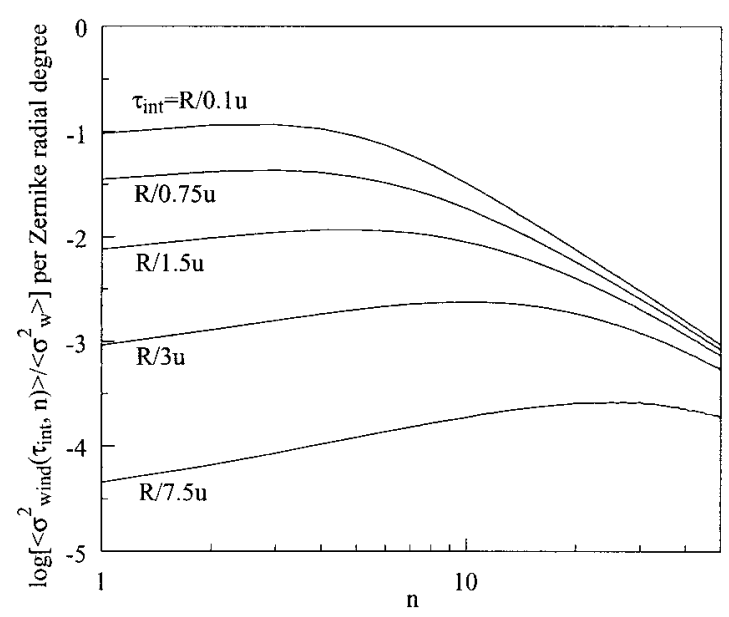

Fig. 3. Wave-front variance that is due to wind-induced deformations on time scales shorter than $\tau_{\text {int }}$, normalized to the total wavefront variance in the range $0.1 u / R \leq f \leq 20 u / R$ and $1 \leq n \leq 50$ for a normal-incidence wind model with outer scale $L_{m}=R$ [Eqs. (25) and (28)]. For $R=15 \mathrm{~m}$ and $u=1 \mathrm{~m} \mathrm{~s}^{-1}$, the frequency range is $0.0067 \leq f \leq 1.33 \mathrm{~Hz}$, and the outer scale is $15 \mathrm{~m}$, which corresponds to a frequency of $0.067 \mathrm{~Hz}$. 
cause $K(N)=K$, where $\mathrm{N}$ is the radial degree of the highest-order deformation, and $K$ is the actuator stiffness, we obtain

$$
\frac{K(n)}{K} \sim \frac{n^{2}}{N^{2}}\left[1-\frac{K(1)}{K}\right]+\frac{K(1)}{K} \text { for } N \gg 1,
$$

where $K(1)$ is the stiffness for the $n=1$ (tip and tilt) modes.

If the wind inside the dome is driven primarily by the shear at the dome opening, the airflow in the dome will be mainly rotation, and the flow at the mirror will be across the surface rather than normal to it. In this case, the appropriate inertial range model is a frozen turbulent screen moving across the mirror surface. This is again a crude model, because it assumes that inhomogeneities in the screen do not evolve as they move across the mirror. The analysis follows Eqs. (14)-(17), but with the $k^{-13 / 3}$ law for the power spectrum of pressure fluctuations. The temporal frequency spectrum of the $j$ th Zernike coefficient is

$$
\begin{aligned}
& \Psi_{j}\left(\frac{f R}{u}\right)=\int_{-\infty}^{\infty} \frac{n+1}{\pi^{2}} \\
& \times \frac{A\left(\mathrm{~J}_{n+1}\left\{2 \pi\left[(f R / u)^{2}+k_{y}{ }^{2}\right]^{1 / 2}\right\}\right)^{2}}{\left\{\left[(f R / u)^{2}+k_{y}{ }^{2}\right]^{13 / 6}+\left(R / L_{m}\right)^{13 / 3}\right\}\left[(f R / u)^{2}+k_{y}{ }^{2}\right]} \\
& \times \begin{cases}2 \cos ^{2} m \phi & \text { even } j \\
2 \sin ^{2} m \phi & \text { odd } j \\
1(m=0)\end{cases}
\end{aligned}
$$

where $\mathbf{u}=(u, 0)$ is now the velocity of the frozen screen. For the $n$th radial degree,

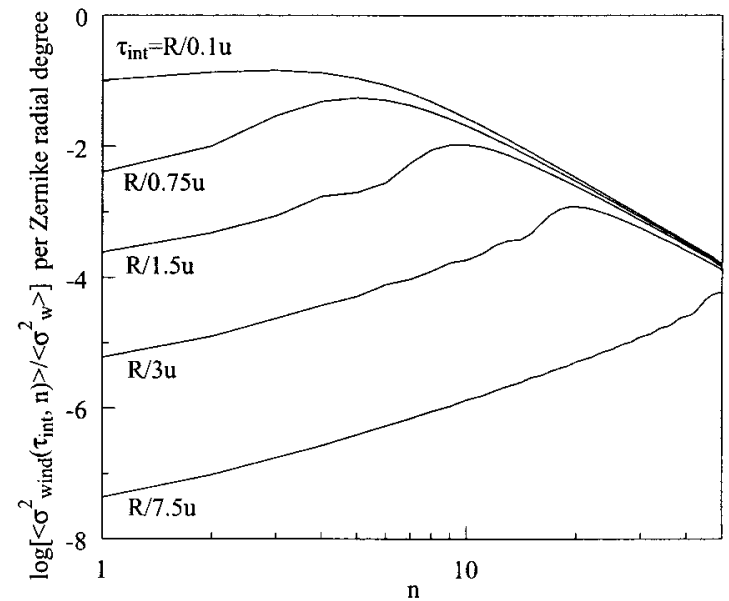

Fig. 4. Same as Fig. 3, but for a frozen screen wind model [Eqs. (33) and (28)].

becomes significant. ${ }^{3}$ Deformation corrections based on the edge sensor readings should therefore be restricted to the higher-order modes.

Adjacent segments are displaced by a distance corresponding to the edge sensor noise, so the total displacement in a particular mode feature is roughly equal to the edge sensor noise multiplied by the number of segments across the radius of the feature. The feature size and the number of segments across the feature are proportional to $1 / n$. For higherorder modes, there are many uncorrelated mode features in the mirror, and the rms segment displacement grows as the square root of the number of features. If the feature size is proportional to $1 / n$,

$$
\Psi_{n}\left(\frac{f R}{u}\right)=\int_{-\infty}^{\infty}\left(\frac{n+1}{\pi}\right)^{2} \frac{A\left(\mathrm{~J}_{n+1}\left\{2 \pi\left[(f R / u)^{2}+k_{y}{ }^{2}\right]^{1 / 2}\right\}\right)^{2}}{\left\{\left[(f R / u)^{2}+k_{y}{ }^{2}\right]^{13 / 6}+\left(R / L_{m}\right)^{13 / 3}\right\}\left[(f R / u)^{2}+k_{y}{ }^{2}\right]} \mathrm{d} k_{y} .
$$

Figure 4 shows the normalized wave-front variance $\left\langle\sigma_{\text {wind }}{ }^{2}\left(\tau_{\text {int }}, n\right)\right\rangle /\left\langle\sigma_{w}{ }^{2}\right\rangle$ for the frozen screen wind model. The power spectrum of the Zernike coefficients is steeper at high frequencies than in the normal-incidence model, so the frozen screen gives a faster decrease in wave-front variance with increasing $\tau_{\text {int }}$. The steeper spectrum in the frozen screen model indicates that the change in the Zernike coefficients for a small lateral displacement of the turbulent field is less than for a small displacement normal to the mirror.

\section{Mirror Control System Noise}

The noise added by the mirror control system depends on the design details, but a simple model can be used to describe the general behavior of systems with edge sensors. The edge sensors are maximally sensitive to modes that have features comparable with the size of a segment, but for low-order modes the sensitivity is poor and the control system noise the number of features in the mirror is proportional to $1 / n^{2}$. For the lowest-order modes, which have a single feature filling the entire mirror, the noise added by the control system is $\sim \delta_{e}(R / d)$, where $\delta_{e}$ is the edge sensor noise and $R / d$ is the number of segments across the mirror radius. The control system noise for a mode with radial degree $n$ is then $\delta_{\mathrm{CS}}(n) \sim$ $\delta_{e}(R / d)\left(1 / n^{2}\right)$. Because there are $n+1$ azimuthal modes in the $n$th radial degree, the wave-front variance contributed by the $n$th radial degree is

$$
\left\langle\sigma_{\mathrm{CS}}{ }^{2}(n)\right\rangle \sim(n+1)\left(2 \frac{2 \pi}{\lambda} \delta_{e} \frac{R}{d} \frac{1}{n^{2}}\right)^{2} .
$$

The edge sensor noise $\delta_{e}$ has contributions from the readout electronics and from manufacturing tolerances in the edge sensor capacitors. On millisecond time scales, the readout noise dominates and $\left\langle\sigma_{\mathrm{CS}}{ }^{2}(n)\right\rangle$ is inversely proportional to the integration time at the sensor output. On longer time scales, 
Table 1. Mirror Control System Noise Model Parameters for the CELT and Keck Telescopes

\begin{tabular}{|c|c|c|c|c|}
\hline \multicolumn{2}{|c|}{ Parameter } & Keck & CELT & Description \\
\hline$M$ & & 108 & 3294 & Number of actuators \\
\hline$N$ & & 13 & 80 & Highest radial degree in mode expansion (see Section 3 ) \\
\hline $\mathrm{R} / d$ & & 4 & 17 & Number of segments across mirror radius (noise multiplier for $n=1$ ) \\
\hline$\left(\frac{\delta_{\mathrm{CS}}(N)}{\delta_{e}}\right)$ & $=\frac{R}{d} \frac{1}{N^{2}}$ & 0.024 & 0.0027 & Noise multiplier for $n=N$ \\
\hline
\end{tabular}

the roughness of the capacitor surfaces and edges becomes important and $\left\langle\sigma_{\mathrm{CS}}{ }^{2}(n)\right\rangle$ is independent of the sensor integration time. Table 1 summarizes the parameters of the Zernike expansion of the control system noise for the CELT and Keck telescopes. This simple model is in good agreement with a more detailed analysis by Chanan et al. ${ }^{3}$

\section{Discussion}

The Zernike expansions of atmospheric phase distortions, wind-induced deformations, and mirror control system noise can be used to design a control strategy that minimizes the mirror surface errors. The loworder mirror modes are corrected with wave-front measurements on a guide star, and $\tau_{\text {int }}$ is adjusted for each mode so that the wave-front variance added to the target, as a result of the mirror being corrected by measurements on the guide star, is equal to the wavefront variance that is due to uncorrected windinduced deformations. The high-order modes are corrected with edge sensor measurements, and the controller time constant is adjusted for each mode so that the wave-front variance that is due to uncorrected wind-induced deformations is less than the wave-front variance that is due to the mirror control system noise. For both the wave-front- and edgesensor-based corrections, the controller time constant generally decreases with increasing $n$. Figure 5 shows an example Zernike mode expansion of the wave-front errors for the parameters given in Table 2 and a normal-incidence wind model. The atmosphere model is for a typical observation at a good site, and the telescope parameters are for the CELT. ${ }^{16}$ In the example, it is possible to reduce the wave-front error that is due to wind-induced deformations by at least an order of magnitude. The required $\tau_{\text {int }}$ varies from $\sim 8$ $\mathrm{s}$ at $n=1$ to $\sim 5 \mathrm{~s}$ at $n=2$, and edge sensor measurements are used to correct modes with $n \geq 3$. The model values for $\left\langle\sigma_{\mathrm{atm}}{ }^{2}\right\rangle$ assume negligible wave-front sensor noise, i.e., a bright guide star. For an ideal Shack-Hartmann wave-front sensor (WFS), the variance of the measured wave-front is $\sim \pi^{2} / p r_{0}{ }^{2}$ for each subaperture, where $p$ is the number of photons per unit area incident on the telescope aperture. ${ }^{7}$ This noise is roughly uniformly distributed over all the sensed modes, so the wave-front variance in the $n$th radial degree is

$$
\left\langle\sigma_{\mathrm{WFS}}^{2}(n)\right\rangle \sim \frac{\pi^{2}}{p r_{0}^{2}} \frac{2(n+1)}{(N+1)(N+2)},
$$

where $N$ is the highest radial degree sensed [giving a total of $(N+1)(N+2) / 2$ modes]. Adding the wavefront sensor noise is equivalent to increasing the atmospheric noise contribution, which increases $\tau_{\text {int }}$ for a particular radial degree and moves the break between wave-front- and edge-sensor-based corrections to a lower mode. Figure 5 shows the wave-front sensor noise for a 14th magnitude spectral-type AO

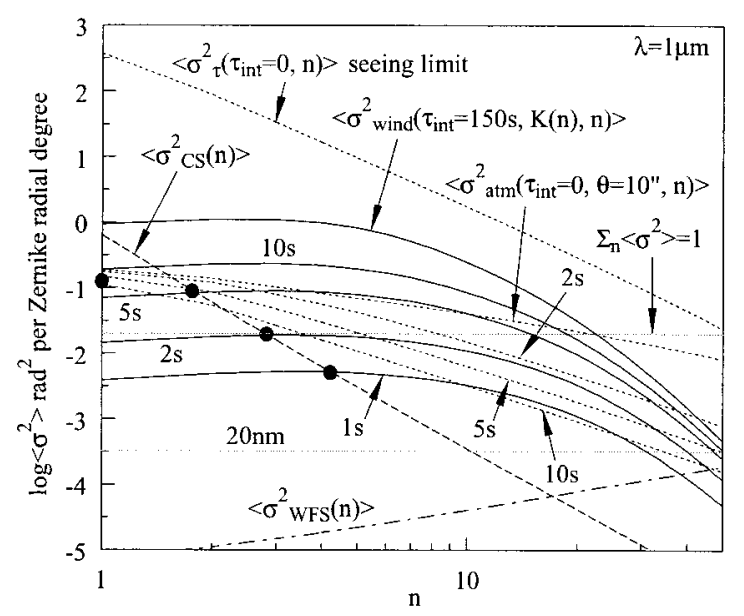

Fig. 5. Contributions to the wave-front variance for $1 \leq n \leq 50$ for the parameters in Table 2 and a normal-incidence wind model [Eq. (25)]. $\left\langle\sigma_{\mathrm{CS}}{ }^{2}(n)\right\rangle$ [approximation (34)] is the wave-front variance contributed by noise in the mirror control system; $\left\langle\sigma_{\text {wind }}{ }^{2}\left[\tau_{\text {int }}, K(n)\right.\right.$, $n]\rangle[$ Eqs. (29) and (30)] is the wave-front variance that is due to wind-induced deformations on time scales shorter than $\tau_{\text {int }}$; $\left\langle\sigma_{\text {atm }}{ }^{2}\left(\tau_{\text {int }}, \theta, n\right)\right\rangle$ [Eqs. (19) and (20)] is the wave-front variance that is added to the target as a result of the mirror being corrected on time scales longer than $\tau_{\text {int }}$ by measurements on the guide star; and $\left\langle\sigma_{\mathrm{WFS}}{ }^{2}(n)\right\rangle$ [approximation (35)] is the wave-front sensor noise for a 14 th magnitude guide star in $0.1 \mathrm{~s} . \quad\left\langle\sigma_{\tau}^{2}\left(\tau_{\text {int }}=0, n\right)\right\rangle$ [Eq. (18)] is the total atmospheric phase distortion in the direction of the guide star, i.e., the seeing limit. $\left\langle\sigma_{\mathrm{atm}}{ }^{2}\left(\tau_{\text {int }}=0, \theta=10 \mathrm{arc} \mathrm{sec}, n\right)\right\rangle$ is the total atmospheric phase distortion on the target, after the atmosphere is corrected in the direction of a guide star 10 arc sec away. This is the seeing after an adaptive correction on the guide star. The line at $\Sigma\left\langle\sigma^{2}\right\rangle=1$ represents a simple model of the diffraction limit, in which a wave-front variance of $1 \mathrm{rad}^{2}$ is uniformly spread across the first 50 radial degrees. Because there are $n+1$ azimuthal modes in the $n$th radial degree, this model corresponds to each azimuthal mode contributing a wave-front variance roughly proportional to $1 / n$. The line labeled $20 \mathrm{~nm}$ represents a similar model, but with 20 -nm total wave-front error. The filled circles indicate the wave-front error contributions after the mirror is corrected by wave-front and edge sensor measurements. 
Table 2. Model Parameters for Figs. 5 and 6

\begin{tabular}{|c|c|c|c|}
\hline Parameter & Value & Units & Description \\
\hline$\theta$ & 10 & $\operatorname{arc~sec}$ & Offset between guide star and target \\
\hline$h$ & 6 & $\mathrm{~km}$ & Height of turbulent atmospheric layer \\
\hline$\gamma$ & 30 & $\operatorname{deg}$ & Zenith angle \\
\hline$\rho$ & $h \theta / R \cos \gamma=0.022$ & & Normalized offset between guide star and target \\
\hline$R$ & 15 & $\mathrm{~m}$ & Mirror radius \\
\hline$r_{0}$ & 40 & $\mathrm{~cm}$ & Fried parameter \\
\hline$v$ & 10 & $\mathrm{~m} \mathrm{~s}^{-1}$ & Wind speed in turbulent atmospheric layer \\
\hline$L_{0}$ & 150 & $\mathrm{~m}$ & Outer scale in turbulent atmospheric layer \\
\hline$\lambda$ & 1 & $\mu \mathrm{m}$ & Wavelength \\
\hline$A_{\text {seg }}$ & 0.2 & $\mathrm{~m}^{2}$ & Segment area per actuator \\
\hline$K$ & $10^{7}$ & $\mathrm{Nm}^{-1}$ & Actuator stiffness \\
\hline$\rho_{\text {air }}$ & 1.29 & $\mathrm{~kg} \mathrm{~m}^{-3}$ & Density of air \\
\hline$u$ & 1 & $\mathrm{~m} \mathrm{~s}^{-1}$ & Wind speed at mirror surface \\
\hline$\sigma_{u} / u$ & 0.5 & & Rms wind speed at mirror surface \\
\hline$f_{m}$ & 0.0067 & $\mathrm{~Hz}$ & Low-frequency limit for wind measurements \\
\hline$L_{m}$ & 15 & $\mathrm{~m}$ & Outer scale in wind at mirror surface \\
\hline$R$ & 17 & & Number of segments across mirror radius \\
\hline \multicolumn{4}{|l|}{$\bar{d}$} \\
\hline$\delta_{e}$ & 3 & $\mathrm{~nm}$ & Edge sensor noise \\
\hline$M$ & 3294 & & Number of actuators \\
\hline$N$ & 80 & & Highest radial degree in mode expansion \\
\hline$T(f)$ & $1-f^{2} /\left(f^{2}+1 / \tau_{\text {int }}{ }^{2}\right)$ & & Control system transfer function \\
\hline$K(n) / K$ & $0.000141 n^{2}+0.1$ & & Mirror stiffness model, $K(1)=0.1 K(80), K(80)=K$ \\
\hline$p$ & 5 & Photons $\mathrm{cm}^{-2} \mathrm{~s}^{-1}$ & $\begin{array}{l}\text { Photon flux on wave-front sensor (14th magnitude } \\
\text { spectral-type AO star, } 0.5-\mu \mathrm{m} \text { bandwidth) }\end{array}$ \\
\hline
\end{tabular}

star and 0.1-s integration time. Even with such a bright guide star, the wave-front sensor noise dominates for measurements at high $n$. Figure 6 shows the example wave-front error analysis, but with a frozen screen wind model. The wind-induced deformations at low $n$ are a little smaller for the frozen screen, but the difference between the two wind models is much less than with the sharp controller transfer function used for Figs. 3 and 4 . For the frozen screen, the required $\tau_{\text {int }}$ is $\sim 20$ s at $n=1$, compared with $\sim 8 \mathrm{~s}$ for the normal-incidence model. For $n>$ 1 , the two models predict similar values of $\tau_{\text {int }}$.

In general, the high-order mirror modes are the

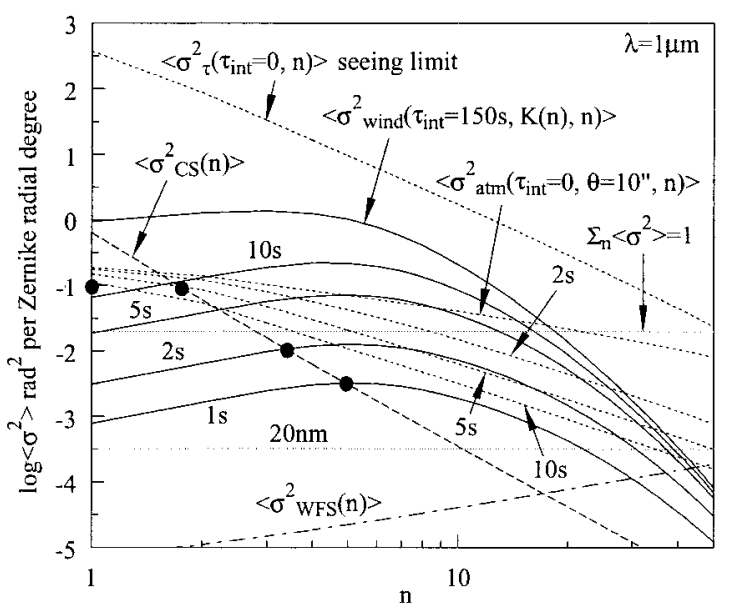

Fig. 6. Same as Fig. 5, but for a frozen screen wind model [Eq. (33)]. most difficult to correct because they change on time scales shorter than a second, but their contribution to the wave-front error is small. The low-order modes are much more important and are fairly easy to correct because they change on time scales of a few seconds. These modes can be corrected by use of an adaptive mirror at the telescope output or by an adjustment of the telescope mirror. The latter approach probably does not require any special hardware because the frequency of the corrections is below any resonances in the telescope structure. In the example, with a 10-arc sec offset between the target and the guide star, the total atmospheric phase distortion on the target, after the atmosphere is corrected in the direction of the guide star, is approximately an order of magnitude smaller than the total wave-front error that is due to wind-induced deformations; so correcting the deformations gives a substantial improvement. For large target to guide star offsets, the atmospheric phase distortion on the target dominates, and wind-induced deformations in the mirror can be ignored.

The author thanks R. Dekany, D. MacMartin, and K. Matthews for helpful comments and suggestions. This research was supported by the Caltech Discovery Fund.

\section{References}

1. F. F. Forbes, "The effects of wind loads on large telescopes and their enclosures," in ESO Conference on Very Large Telescopes and their Instrumentation, ESO Rep. 30, M.-H. Ulrich, ed. (European Southern Observatory, Garching, Germany, 1988), pp. 831-843. 
2. J. E. Nelson, T. S. Mast, and S. M. Faber, eds., Design of the Keck Observatory and Telescope, Keck Observatory Rep. 90 (Keck Observatory, Kamuela, Hawaii, 1985).

3. G. Chanan, J. E. Nelson, C. Ohara, and E. Sirko, "Design issues for the active control system of the California Extremely Large Telescope (CELT)," in Telescope Structures, Enclosures, Controls, Assembly/Integration/Validation and Commissioning, T. Anderson, ed., Proc. SPIE 4004, 363-372 (2000).

4. D. G. MacMartin, T. S. Mast, G. Chanan, and J. E. Nelson, "Active control issues for the California Extremely Large Telescope," presented at the AIAA Guidance, Navigation, and Control Conference, Montreal, 6-9 Aug. 2001 (American Institute of Aeronautics and Astronautics, Reston, Va., 2001), paper AIAA-2001-4035.

5. R. J. Noll, "Zernike polynomials and atmospheric turbulence," J. Opt. Soc. Am. 66, 207-211 (1976).

6. F. Roddier, M. J. Northcott, J. E. Graves, and D. L. McKenna, "One-dimensional spectra of turbulence-induced Zernike abberations: time-delay and isoplanacity error in partial adaptive compensation," J. Opt. Soc. Am. A 10, 957-965 (1993).

7. F. Roddier, ed., Adaptive Optics in Astronomy (Cambridge University, Cambridge, UK, 1999), Chap. 3.

8. F. Roddier, "The effects of atmospheric turbulence in optical astronomy," in Progress in Optics, E. Wolf, ed. (North-Holland, Amsterdam, 1981), Vol. 19, pp. 281-376.

9. V. I. Tatarskii, The Effects of the Turbulent Atmosphere on Wave Propagation (U.S. Department of Commerce, Springfield, Va., 1971).

10. D. L. Fried, "Statistics of a geometric representation of wavefront distortion," J. Opt. Soc. Am. 55, 1427-1435 (1965).

11. A. Ishimaru, Wave Propagation and Scattering in Random Media (Academic, New York, 1978), Vol. 2.

12. J. W. Hardy, Adaptive Optics for Astronomical Telescopes (Oxford University, Oxford, UK, 1998), Chap. 3.

13. N. Roddier, "Atmospheric wavefront simulation using Zernike polynomials," Opt. Eng. 29, 1174-1180 (1990).

14. M. K. Cho, L. M. Stepp, and S. Kim, "Wind buffeting effects on the Gemini 8m primary mirrors," in Optomechanical Design and Engineering 2001, A. E. Hatheway, ed., Proc. SPIE. 4444, 302-314 (2001).

15. S. Timoshenko and S. Woinowsky-Krieger, Theory of Plates and Shells (McGraw-Hill, New York, 1959), Chap. 3.

16. T. S. Mast and J. E. Nelson, "Segmented mirror control system hardware for CELT," in Optical Design, Materials, Fabrication and Maintenance, P. Dierickx, ed., Proc. SPIE 4003, 226-240 (2000). 